\title{
Elicited Imitation in Lexical Development: Evidence from a Study of Temporal Reference
}

\author{
Deborah Keller-Cohen ${ }^{1,2,3}$
}

Accepted March 10, 1979

This study examined the use of elicited imitation in investigating lexical development within a semantic domain. For this purpose the acquisition of reference to sequence and simultaneity by 3-5 year old children was examined. Three factors were proposed to account for the order in which lexical items within a semantic field are acquired: restrictedness of a lexical item, congruence with perceptual strategies, and conceptual simplicity. A significantly greater number of correct responses was found in sentences describing sequential events than in simultaneous events. Furthermore, imitations of sentences referring to simultaneity were more degraded than imitations of sentences referring to sequence. The children seemed to begin acquiring reference to temporally related events by learning about words describing serially ordered events. A three stage developmental model is proposed to account for the results.

\section{INTRODUCTION}

Recent psycholinguistic research has been concerned with the ways in which children acquire lexical items. Studies of spontaneous speech and

\footnotetext{
'Department of Linguistics and Center for Human Growth and Development, University of Michigan, Ann Arbor, Michigan 48109.

2 Address correspondence to Dr. Deborah Keller-Cohen, Department of Linguistics, University of Michigan, Ann Arbor, Michigan 48109.

${ }^{3}$ Some of the data reported here are drawn from the author's doctoral dissertation from the Department of Linguistics, State University of New York, Buffalo, 1974.
} 
tests of comprehension have been principle means of unraveling clues to how children build their lexicons (Bowerman, 1977; Klatzky et al., 1973; Wilcox and Palermo, 1974/1975). Tests of elicited imitation however, have rarely been used to investigate the acquisition of lexical items in a semantic field.

In elicited imitation, a child is asked to repeat a model sentence immediately after it has been produced by an experimenter. It is thought that when the sentence is too long or complex to reproduce by rote, the child makes systematic errors repeating the model sentence (Menyuk, 1963; Labov et al., 1968). Slobin and Welsh (1973) termed these errors assimilatory deformations since they argued that the child assimilates the stimulus material, recoding and restructuring it to be congruent with what he knows about his language.

Because elicited imitation has rarely been used to investigate lexical development, there has been no systematic discussion of the kind of information it might provide about the acquisition of words. Children's imitations of lexical items can be evaluated in two ways: accuracy of the imitation and content of the imitation. If the sentences to be imitated exceed the child's short term memory, examining which words children imitate accurately should offer a picture of the terms of reference they have acquired. Evaluating the content of a child's imitation is more problematic. For example, when a child substitutes one word for another, there is no way to determine what meaning he has assigned the substitution. Even so, substitutions may suggest which terms a child has available within a semantic field. Considering the terms which actually appear in the imitations of children at different ages should provide a clearer picture of the order in which terms enter a semantic domain. Furthermore, differences between individual children in patterns of substitution should provide a clearer picture of particular paths in the development of a semantic domain.

What factors might influence the acquisition of terms within a semantic field? Prior research suggests that several factors interact to explain order of acquisition within a field. (1) Restrictedness-in some semantic fields, the least restricted lexical items are acquired first. For example, among dimensional adjectives (e.g., big-small, tall-short) the least restricted terms, big and small, are learned first (Bartlett, 1976; Wales and Campbell, 1970). Whereas big and small can be used to refer to one, two, or three dimensions, other dimensional adjectives do not share this flexibility. (2) Congruence-children often first learn terms which describe relations that are congruent with the perceptual strategies they employ. For example, children pay attention to movement toward them 
rather than away and this strategy is reflected in their acquisition of lexical items. Clark and Garnica (1974) observed the acquisition of come prior to go and Garman et al. (1970) found to learned earlier than from. (3) Conceptual simplicity-conceptual simplicity has a variety of meanings. At least one way in which terms may differ in complexity is in the nature of the relations underlying use of the terms. If the relation which one term describes requires a child to make fewer discriminations than some other term, the former can be regarded as conceptually simpler. For example, reference to one point in time (today, now) is learned earlier than reference to two points in time ( $X$ and then $Y$ ) (Clark, 1973). Determining when one event occurs requires fewer judgments than determining the time of two points and their relation.

How might these factors influence the acquisition of lexical items that temporally relate actions? Consider the following terms: and, first, last, before, after, while, at the same time. And ought to be the term learned first on three accounts: it is the least restricted, making reference to nontemporal relations, sequentially order events, and simultaneous events; it also permits descriptions that are congruent with children's sequential processing of events (Piaget, 1969); and it is conceptually simpler since it does not require the child to clearly establish the temporal relation between two events.

First, last, before, and after are more restricted than and since they describe serially ordered events only. These terms describe temporal relations that are congruent with the child's sequential processing of two events and, therefore, should not present difficulties in this respect. They are more complex conceptually than and since the child must have established a temporal relation between two events to use these words.

While and at the same time share some referential restrictions similar to before and after since they describe one temporal relation only. Like the terms that refer to sequence, while and at the same time also require a child to establish a temporal relation whereas and does not. Unlike words describing sequential relations, while and at the same time make reference to relations that are incongruous with the child's strategy for perceiving temporally related events sequentially. Hence, they ought to be learned later than before and after for example.

Previous research supports the order of acquisition sketched above. In a cross-cultural study of spontaneous speech, Clancy et al. (1976) found and the first temporal term to be learned, preceding before and after. In a study of children's comprehension, Keller-Cohen (1974) found before and after understood earlier than at the same time and while.

The central aim of this paper was to explore whether elicited 
imitation could provide a view of how a child builds up a system of reference within a semantic field. A second issue considered was the effect of lexical items on sentence processing. Bloom et al. (1975) found that one factor that constrains utterance length is the presence of a newly learned lexical item. Apparently the allocation of resources to use such elements results in utterances that are less complex than the child is otherwise capable of producing. Elicited imitation provides a unique opportunity to explore this experimentally. If a child must repeat a sentence with a lexical item he has not fully analyzed, he ought to produce a more degraded imitation than sentences with lexical items he has analyzed more fully.

The last issue explored is the possible influence of aspect on the child's acquisition of temporal reference. In conjunction with a temporal connective, the English progressive is often used to signal simultaneity between two events whereas the past may be used to indicate completion (Leech, 1971). Although little work examines the interaction between temporal connectives and verb forms in language acquisition, available evidence suggests that children below 5 years of age have not acquired the completive-continuation contrast between the past and the progressive verb forms (Feagans, 1974). In light of these findings, it was predicted that aspect would not affect a child's interpretation of temporal connectives until nearly 5 years of age. It seemed that at this age the acquisition of the progressive might aid a child in interpreting sentences describing simultaneity since he would have begun to be sensitive to both the verb form and the temporal connective.

In the report to follow, elicited imitation was used to examine the acquisition of terms that temporally relate actions. Sentences of different structural composition (transitives and intransitives) were included to provide empirical support for Slobin and Welsh's claim that utterance length influences performance in elicited imitation.

On the basis of the discussion above five major predictions were made: (1) correct imitations should be more frequent in response to sentences referring to sequence than in response to those describing simultaneity; (2) imitations that are more degraded were expected in response to sentences describing simultaneous events than those describing sequential events; (3) more accurate imitations were expected on the shorter, structurally less complex intransitive sentences than the transitives; (4) a three-stage developmental sequence was predicted based on the distribution of both correct imitations and substitution responses: at the first level, children will use only the least restricted term (and) and 
will be poor at imitating all sentences. At the second level, children will use and plus some terms from the next most restricted set, those referring to sequence. They will correctly imitate some sentences referring to sequence, but do poorly on sentences describing the simultaneity of two different events. The third level will consist of children who use terms from the first two sets and some from the third set, words describing simultaneity. They will do well on sentences referring to sequence and their performance on simultaneity will be improved over that of level 2 children; (5) near 5 years of age, aspect was expected to effect performance on the imitation of sentences describing simultaneity. Sentences with verbs in the past progressive were expected to elicit better performance on simultaneity than those with verbs in the simple past.

\section{METHOD}

\section{Subjects}

A total of 32 children ( 16 boys, 16 girls) were drawn from the Greater Buffalo area, 29 from the Country Day Nursery School, and 3 from outside the school. The latter three were necessary to meet the age and sex requirements of the design. They were middle class children and all were native speakers of English, although one spoke Hungarian and English bilingually. The subjects were partitioned into 4 age groups: I: 2;11-3;5 ( $\bar{x}=3 ; 2)$; II: $3 ; 9-4 ; 2 \quad(\bar{x}=4 ; 0)$; III: 4;3-4;6 $(\bar{x}=4 ; 5)$; IV: $4 ; 7-4 ; 11(\bar{x}=4 ; 10)$.

\section{Instrument}

Nine temporal constructions in five temporal categories were examined. Examples of the constructions in test sentences appear below.

1. First the boy pulled the cup. Last the girl hit the mouse.

2. The boy was jumping over the box and then the girl was pushing the dog.

3. The girl kicked before the boy hopped.

4. After the boy pet the cat, the girl pushed the cup.

5. Before the girl was pushing the elephant, the boy was pulling the box.

6. The boy was kicking the car after the girl was kissing the dog. 
7. The girl was skipping and the boy was jumping at the same time.

8. The boy was hopping over the cup while the girl was pushing the shoe.

9. The girl and boy threw the box together.

Each construction appeared four times and each subject received a randomized presentation of the sentences.

In (1)-(4) the order of the clauses is the same as the temporal order of the events. These will be referred to as simple sequentiality (SSE). First, Last, and and then will be termed $\mathrm{SSE}_{1}$ and order of mention before and after $\mathrm{SSE}_{2}$ to distinguish conjuncts $\left(\mathrm{SSE}_{1}\right)$ from subordinators $\left(\mathrm{SSE}_{2}\right)$. In (5) and (6) the clause order is the reverse of the temporal order of the events. These will be termed reverse sequentiality (RSE). Sentences (7) and (8) express the simultaneity of two distinct events (SIM) where the clause order does not correspond in any way to the temporal order. Sentence (9) describes the participation of two actors in one action at one point in time. This construction will be termed single point simultaneity. This construction was considered a separate temporal category since the actions expressed in together sentences are not separable as are the actions in (7) and (8). In all, five temporal categories were examined in this study ( $\mathrm{SSE}_{1}, \mathrm{SSE}_{2}, \mathrm{RSE}, \mathrm{SIM}$, together). Part 1 of each test consisted of transitive sentences and Part 2 contained intransitives. Each clause contained the boy or the girl as the subject, a verb (jump over, hop over, push, pull, kick, hit, kiss, pet, or throw) and all transitive sentences contained an object (mouse, dog, cat, elephant, flower, rock, shoe, box, or cup). The presentation of the two parts was counterbalanced across age and sex.

Aspect was a between subject variable. One-half of all subjects (an equal number of males and females per age group) heard all test sentences in the past progressive (e.g., was pushing) and one-half heard sentences in the simple past (e.g., pushed). In sum, age and aspect were between subject variables and sentence complexity and temporal category was within subject variables.

\section{Procedure}

Each subject was tested individually in a room adjacent to the classrooms, and the responses of each child were tape-recorded. Each child was told that he was going to play a game. $E$ asked the child to listen to what she said and to "say what I say." Two intransitive practice sentences were presented to ensure that the instructions had been understood. An attempt was made to read each sentence twice only. 
However, in the case of inattentive or distracted subjects, additional readings were given. In light of the theoretical assumptions underlying this study, it was felt that additional readings would not alter the subject's understanding of the target phenomena. Part 1 and Part 2 of the test were administered in separate sessions for each subject.

All data were transcribed by $E$. To determine reliability, a second judge transcribed $13 \%$ of the data. Four protocols, one from each age group, were randomly selected. For every test sentence, the two transcripts were compared on each of the following sentence elements in both clauses: the definite article, the subject, the verb, the verb aspect, the definite article and the object for transitive sentences, and the temporal connective. The two sets of transcriptions agreed on $93 \%$ of the comparisons.

\section{Scoring}

Each imitation was assigned a rank score from 1-7 points with 7 points for a perfect imitation and 1 point for no response or an entirely incorrect imitation. The purpose of the ranking was to examine the effect of lexical items on the processing of other elements in the model sentences. The scoring scale with examples appears below.

7-Perfect imitation.

6-The stimulus sentence is imitated correctly, but the temporal relationship is expressed in a synonymous lexical form.

5-The temporal connective is reproduced correctly or synonymously, but the remainder contains no more than one lexical error and one grammatical error.

4-The temporal connective is reproduced correctly or synonymously, but the remainder contains several lexical or grammatical errors. Furthermore, in each clause at
The boy threw the ball before the girl kicked the shoe.

After the boy threw the ball the girl kicked the shoe.

The boy threw the ball before the girl hit the shoe. The boy was throwing the ball before the girl kicked the shoe.

The boy hit the ball before the girl was throwing the dog. 
least two of the major constituents (SVO) had to be correctly imitated (SV, VO, or SO). In intransitives, at least 1 of the major constituents had to be correctly imitated.

3-The two SVO sequences are correctly reproduced, but the connective is either incorrect or absent.

The boy threw the ball, the girl kicked the shoe. / The boy threw the ball after the girl kicked the shoe.

2-One SVO sequence is imitated correctly. The connective may be incorrect or abThe boy threw the ball. / The boy threw the ball when the boy dropped the car. sent and the other SVO sequence may be incorrectly imitated or absent.

1-No response or everything is The boy hit her. incorrectly imitated.

And and when substitutions were considered incorrect in response to both sequence and simultaneity since they could not be interpreted unambiguously. And in place of and at the same time and and then was also considered incorrect. This coding scheme provided a particularly stringent test of the hypotheses because and substitutions were nine times more frequent in response to sequential sentences than in response to simultaneous sentences. However, prior research could have been used to justify scoring as correct all and imitations in response to sequence. Feagans (1974) found that even by 5 years of age, the modal interpretation of sentences with and is sequential.

The four protocols used to determine interrater reliability on transcribing were used to determine coding reliability. Scoring by $E$ and the judge agreed on $92 \%$ of these sentences.

\section{RESULTS}

In the first analysis, each subject received a total point score for each of the five temporal categories. The points for each subject were calculated by multiplying the number of responses by the rank value (1-7) 
achieved per response, with a maximum of 28 points per temporal category possible. A repeated measures analysis of variance (sentence complexity $\times$ aspect $\times$ age $\times$ temporal category) revealed a significant main effect for age: $F(3,24)=14.48, p<0.001$. The oldest children (Group IV) achieved a mean score of 21.4 points and the youngest children (Group I) 12.9 points.

The age groups were found to differ significantly on their imitation of aspect, $F(3,24)=4.36, p<0.05$. Performance on the simple past improved more rapidly than performance on the past progressive. In addition, performance on intransitive sentences was found to be superior to that on transitives. $F(1,24)=10.88, p<0.001$. The interaction between temporal category and aspect was nonsignificant, $F(4,96)=0.30$.

The effect of temporal construction was found to be reliable, $F(4,96)$ $=74.9, p<0.001$. Table I reports the proportion of correct responses (ranks 4-7) by age in each temporal category. The children were more likely to imitate correctly sentences referring to sequence than those referring to two point simultaneity. Performance on the temporal categories from best to worst was: (1) together; (2) $\mathrm{SSE}_{2}$; $\mathrm{SSE}_{1}$; (3) RSE; (4) SIM. Scores on RSE were lower than on SSE, $t(31)=2.96$, $p<0.005$. As predicted, the temporal constructions eliciting the fewest correct responses were those expressing the simultaneity of two different events. Performance was equally poor on both at the same time and while, $t(31)=-0.27$. The hypothesis that reference to sequence is learned earlier than reference to simultaneity was confirmed by significantly higher scores on RSE than on SIM, $t(31)=3.22, p<0.005$.

Table I. Proportion of Correct Responses by Age for Each Temporal Category

\begin{tabular}{lccccc}
\hline & \multicolumn{5}{c}{ Temporal category } \\
\cline { 2 - 6 } Age & SSE $_{1}$ & SSE$_{2}$ & RSE & SIM & Together \\
\hline Group I & 0.41 & 0.42 & 0.37 & 0.31 & 0.76 \\
Group II & 0.48 & 0.55 & 0.49 & 0.41 & 0.83 \\
Group II & 0.58 & 0.66 & 0.60 & 0.55 & 0.92 \\
Group IV & 0.73 & 0.80 & 0.74 & 0.63 & 0.92 \\
\hline
\end{tabular}




\section{Error Analysis}

The group results reported above do not reveal individual patterns of response used. To obtain a view of this, the children were first sorted into 2 groups: those with $\geq 75 \%$ correct on both SEQ and SIM [Pattern A] and those with $<75 \%$ correct on both SEQ and SIM. The former group consisted of 6 children, the latter 26 .

Of the 26 children who did not reach criterion on both temporal categories, 7 did not use any of the temporal terms presented in $75 \%$ of their responses. Three of these 7 produced imitations with one clause only or two clauses with no connectives [Pattern B].

\section{Pattern B}

1. A girl pushed a box. The girl was kissing the mouse.

2. The girl was petting the mouse, the boy was pulling the car. The boy was kissing the dog, the girl pulled the box.

The modal response of the remaining 4 out of 7 children was two clauses connected by and [Pattern $\mathrm{C}$ ].

\section{Pattern C}

The boy was petting the elephant and the girl was hitting the cat.

The boy did summersaulted and the girl did kick.

The remaining 19 children who did not reach criterion on both temporal categories were sorted into three groups by pattern of response: 9 children reached criterion on SEQ only [Pattern D], 2 on SIM only [Pattern E], and 8 produced a mixed pattern of responses [Pattern $\mathrm{F}$ ]. These last 8 did not reach criterion on either SEQ or SIM. The number of children by age displaying these responses appears in Table II.

Of the nine children who reached criterion on SEQ only [Pattern D], four substituted when for while in at least $75 \%$ of their imitations of while (three of four responses). This was not true of the remaining five. In fact, of these children only one used when in response to while and it appeared only once.

The two children who reached criterion on SIM only [Pattern E] used all the SEQ terms in the model sentences, but made errors in their imitations of these terms. Nothing systematic could be said of their errors.

The remaining eight children who produced a mixture of responses, 
Table II. Modal Response Patterns by Age

\begin{tabular}{rcccccc}
\hline Age group & $\begin{array}{c}\text { Nonrelational } \\
\text { or omissions }\end{array}$ & and & Mixture & $\begin{array}{c}\text { Criterion } \\
\text { on SEQ only }\end{array}$ & $\begin{array}{c}\text { Criterion } \\
\text { on SIM only }\end{array}$ & $\begin{array}{c}\text { Criterion } \\
\text { on SEQ } \\
\text { and SIM }\end{array}$ \\
\hline I & 2 & 3 & 2 & 0 & 0 & 1 \\
II & 1 & 1 & 1 & 3 & 1 & 1 \\
III & 0 & 0 & 3 & 2 & 1 & 2 \\
IV & 0 & 0 & 2 & 4 & 0 & 2 \\
\hline & 3 & 4 & 8 & 9 & 2 & 6 \\
\hline
\end{tabular}

Pattern F, could be characterized as follows: four achieved $\leq 50 \%$ correct on both SEQ and SIM; three had $>50 \%$ correct on SEQ, but not SIM; one had $>50 \%$ correct on both SEQ and SIM. So, if a child performed above chance on only one temporal category, it was likely to be SEQ.

\section{DISCUSSION}

The evidence presented here supports the view that when stimulus sentences in elicited imitation are sufficiently long and complex, the child actively processes the stimulus material, providing a recoded version that is congruent with his current linguistic knowledge. The findings reported here also support the view that the semantic content of terms to be imitated influences performance. Systematic changes in the accuracy of response were found to depend on the subarea of temporal reference. So sentences of equal length, but different semantic content, elicited different levels of performance. In addition, children made more errors in imitating the entire sentence when the sentence contained a more complex connective. Finally, the systematic changes in substitution responses provided further support for the active encoding of material in elicited imitation. Children seem to select from their pool of avaliable lexical items to produce their imitation responses.

\section{The Acquisition of Temporal Reference}

The picture of temporal reference revealed here in elicited imitation is similar to that found elsewhere. Previous research reports that and is 
the first temporal connective acquired (Clancy et al., 1976; Clark, 1973). The finding that at 3 years of age and was the modal substitution in response to all temporal categories conforms to these earlier results. The greater number of correct imitations of simple sequence than reverse sequence is congruent with studies of comprehension (Clark, 1971; Johnson, 1975). Furthermore, superior performance on sequence in contrast to simultaneity corresponds to comprehension data reported in Keller-Cohen (1974) and Feagans (1974).

The one term all the children seemed to know was together. No child scored less than $88 \%$ correct on this item. One explanation for this is that together is not used to describe two independent events, in fact, it describes the relationship between two participants in one event. In this respect, it is linguistically simpler than the other words investigated. From another point of view, together may not be a part of the child's temporal field at all. Reports from the children's preschool teachers indicate that together is a word introduced quite early at school to encourage cooperation among peers. The teachers reported that the children were frequently requested to play, sit, clap, or sing together for example. In either case, the data do not distinguish between these interpretations and together could not be included in the proposed three-stage developmental model.

\section{The Development of Relational Terms in a Semantic Field}

The data from elicited imitation presented here offer a general view of the way lexical development takes place within a semantic field. Moreover, this is consistent with research reported elsewhere employing different methodologies. For example, it seems that children initially learn to make reference to a set of relations without using overt reference. Apparently, this is accomplished by the juxtaposition of two propositions such as Christy sweater, cold (Bowerman, 1974) or I can't do it, I not big enough (Clancy et al., 1976). In elicited imitation, responses of this sort, that is juxtapositions of two propositions, were found mainly in data from three 3 year olds. They repeated model sentences without any connective, e.g., The girl was kicking, the boy was walking. To what extent those children had any sort of knowledge about temporal connectives cannot be determined from these data. Also observed only among the 3 year olds were children whose modal response was an imitation with and, the most general English connective. Nearly half of all 3 year olds ( 7 of 16) used either of these two patterns of responding. The data suggest that the 
earliest stages of lexical acquisition within a semantic field are: (1) implicit reference (juxtaposition); (2) inexplicit reference (and).

The children's response patterns point to several possible routes following these early stages, although the data are not unequivocal. One possible path is to begin learning terms in one subfield such as reference to sequence, but no terms in another subfield such as reference to the simultaneity of two different events. Evidence for this would be children who produced correct imitations in response to sentences describing serial events and incorrect imitations of sentences referring to simultaneous events (or the reverse). At no age were there children who gave all correct imitations of sequence and none for simultaneity. However, there were children who produced some correct reproductions of sentences describing sequential events, but no correct imitations of sentences describing simultaneous events. In general, correct imitations of sentences referring to serial events surpassed those in response to simultaneity. This would suggest that a child may undertake working out the details of some subfield earlier than those in another; yet it appears unlikely that any child would learn all the terms for reference in one area of a semantic field without any progress in other areas.

One alternate explanation for the apparent later acquisition of while and at the same time is syntactic. Both these constructions were investigated here in sentences with the main clause first. It may be that temporal connectives in sentences with the main clause first are less salient. If this is so, it ought to have been particularly apparent in the children's reproductions of the longer transitive sentences since the length coupled with the connective appearing in noninitial position ought to have led to poor performance. The number of correct responses (ranks 4-7) in sentences with the main clause first (and then, $X$ before $Y, X$ after $Y, X$ while $Y, X$ and $Y$ at the same time) were compared with sentences with the subordinate clause first (After $X Y$, Before $Y X$ ). Sentences with together were excluded from this analysis since they do not contain two clauses. Performance on main clause first sentences such as those with at the same time was significantly better than on sentences with the subordinate clause first, $t(31)=4.1, p<0.001$.

Furthermore, inferior performance on sentences describing simultaneous events cannot be due to the effect of length in sentences with at the same time. If length were critical, performance on while should have been superior to that on at the same time whereas no difference was found. Similarly, if length was a key factor, error rates on while, before, and after, should have been similar; however, performance on while was significantly inferior. 
Another possible explanation for the developmental sequence reported here comes from studies of early lexical development. Huttenlocher (1974) suggested that children know more about words than their spontaneous speech would indicate. Given the demands of spontaneous speech, children may experience difficulty retrieving a particular word. As a result, they may select a word from the same semantic field with which they are more familiar. Applying this to elicited imitation, a child might know a word in a model sentence, but would have difficulty retrieving it because of the performance requirements of the task. As such, lower performance on SIM would be the result of a retrieval rather than a comprehension problem. However, this explanation for lower performance in response to sentences with while and at the same time would be congruent with the interpretation already offered since it would still support the claim that children are more familiar with terms describing serially ordered events.

A three stage developmental model was predicted based on the interaction of three factors: restrictedness, congruence with nonlinguistic strategies, and conceptual simplicity. The data reported here provide support for this. The youngest children who included temporal connectives in their responses selected either and or a term referring to sequence. And is the least restricted temporal term and the terms referring to sequence display congruence with their perceptual strategy of processing events in their order of mention. Seldom did a term describing simultaneous relations appear in their responses. Children between $31 / 2$ and $4 \frac{1}{2}$ years displayed greater success imitating terms describing sequence than simultaneity although there were two children who had apparently worked out the details of while and at the same time sooner than terms such as before and after. However, for nine children, correct imitations of words describing sequential relations came in earlier than terms describing simultaneity as compared to two children who reached criterion on simultaneity first. These findings lend support to the role of a complex of factors in the acquisition of lexical items in a semantic field.

It is also interesting to note that if a child substituted when for a target item, the substitution appeared either in response to SEQ or to SIM, but never in response to both. This suggests that the meaning of a term with multiple readings may at first be restricted to one sense until a child becomes more certain about its use. It also points out that children may differ in their initial interpretation of such items.

This study was aimed at exploring the sorts of information elicited imitation provides about the growth of a semantic field. Although elicited 
imitation cannot provide clear clues to the interim meanings children have for words, it does offer a view of the approach children take to building up semantic domains. Differences between children seem to reflect alternate routes to learning semantically related words. Whether the course of development sketched here is representative of all relational terms will require further research.

\section{REFERENCES}

Bartlett, E. J. (1976). Sizing things up: The acquisition of the meaning of dimensional adjectives. $J$. Child Language, 3:205-219.

Bloom, L., Miller, P., and Hood, L. (1975). Variation and reduction as aspects of competence in language development. In Pick, A. (ed.), Minnesota Symposia on Child Psychology (Vol. 9), University of Minnesota Press, Minneapolis, Minn.

Bowerman, M. (1974). Learning the structure of causative verbs: A study in the relationship of cognitive, semantic and syntactic development. In Papers and Reports on Child Language Development, 8:142-178.

Bowerman, M. (1977). The structure and origin of semantic categories in the language learning child. Paper prepared for the Burg Wartenstein Symposium No. 74, Wenner-Gren Foundation for Anthropological Research.

Clancy, P., Jacobsen, T., and Silva, M. (1976). The acquisition of conjunction: A cross-linguistic study. In Papers and Reports on Child Language Development, 12:71-80.

Clark, E. V. (1971). On the acquisition of the meaning of before and after. J. Verb. Learn. Verb. Behav. 10:266-275.

Clark, E. V. (1973). How children describe time and order. In Ferguson, C. A., and Slobin, D. I. (eds.), Studies of Child Language Development, Holt, Rinehart and Winston, New York.

Clark, E. V. and Garnica, O. K. (1974). Is he coming or going? On the acquisition of deictic verbs. J. Verb. Learn. Verb. Behav. 13:559-572.

Feagans, D. L. V. (1974). The comprehension by young children of some temporal and spatial linguistic structures. Natural Language Studies, Vol. 18, University of Michigan Phonetics Laboratory, Ann Arbor, Mich.

Garman, M. A., Griffiths, P. D., and Wales, R. I. (1970). Murut (Lun Buwang) prepositions and noun particles in children's speech. Sarawak Mus. J. 18:353-376.

Huttenlocher, J. (1974). The origins of language comprehension. In Solso, R. L. (ed.), Theories in Cognitive Psychology, Lawrence Erlbaum Associates, Potomac, Md.

Johnson, H. L. (1975). The meaning of before and after for preschool children. J. Exp. Child Psychol. 19:88-99.

Keller-Cohen, D. (1974). Cognition and the acquisition of temporal reference. In Papers from the Tenth Regional Meeting of the Chicago Linguistic Society, 3:310-320.

Klatzky, R. L., Clark, E. V. and Macken, M. (1973). Asymmetries in the acquisition of polar adjectives: Linguistic or conceptual? J. Exp. Child Psychol. 16:32-46. 
Labov, W., Cohen, P., Robins, C., and Lewis, J. (1968). A study of the non-standard English of Negro and Puerto Rican speakers in New York City, Vol. 1. Columbia University Cooperative Research, New York, New York, Res. Rep. no. 3288.

Leech, G. N. (1971). Meaning and the English Verb, Longman, London, England.

Menyuk, P. (1963). Some effects of grammatical transformations on the recall of English sentences. J. Verb. Learn. Verb. Behav. 2:346-351.

Piaget, J. (1969). The Child's Conception of Time, Ballantine Books, New York.

Slobin, D. I., and Welsh, C. A. (1973). Elicited imitation as a research tool in developmental psycholinguistics. In Ferguson, C. A. and Slobin, D. I. (eds.), Studies of Child Language Development, Holt, Rinehart and Winston, New York.

Wales, R. J., and Campbell, R. N. (1970). The development of comparison and the comparison of development. In Flores d'Arcais, G., and Levelt, W. J. H. (eds.), Advances in Psycholinguistics, North-Holland Publishing, Amsterdam.

Wilcox, S., and Palermo, D. S. (1974/1975). 'In', 'on', and 'under' revisited. Cognition, 3:245-254. 